\title{
SÍNTESE DE ENAMINONAS
}

Helena M. C Ferraz* e Fernando L. C. Pereira

Departamento de Química Fundamental, Instituto de Química, Universidade de São Paulo, CP 26077, 05513-970 São Paulo - SP

Recebido em 18/9/02; aceito em 13/6/03

\begin{abstract}
SYNTHESIS OF ENAMINONES. The general term "enaminone" is applied to any compound bearing the conjugated system N$\mathrm{C}=\mathrm{C}-\mathrm{C}=\mathrm{O}$. Enaminones are typical capto-dative ethylenes, showing particular properties due to $\pi$-electron delocalization. Their reactivity can be predicted by structural patterns of substitution, conformation and configuration, making them versatile synthetic building blocks. In this review, a variety of methods for the synthesis of enaminones is described. The methods were divided into condensation of amines to $\beta$-dicarbonyl compounds, addition of amines to $\alpha, \beta$-unsaturated carbonyl compounds, condensation between carbonyl compounds and $\mathrm{N}$-unsaturated functions and other methods, covering the period from 1993 to 2001.
\end{abstract}

Keywords: $\beta$-enamino ketones; $\beta$-enamino esters; methods of preparation.

\section{INTRODUÇÃO}

O termo geral "enaminona" refere-se a qualquer composto que apresente o sistema conjugado $\mathrm{N}-\mathrm{C}=\mathrm{C}-\mathrm{C}=\mathrm{O}$. Enaminonas são compostos $\beta$-enamino carbonílicos, derivados de $\beta$-dicetonas, $\beta$-ceto ésteres e outros compostos $\beta$-dicarbonílicos. Os representantes mais comuns desta classe são $\beta$-enamino cetonas e $\beta$-enamino ésteres, também chamados de amidas vinílogas e carbamatos ou uretanas vinílogas, respectivamente; denominações como " $\beta$-aminoenona", "acilvinilamina" e "acilenamina" também são encontradas na literatura.

Compostos deste tipo são considerados etilenos capto-dativos típicos, devido ao efeito mesomérico dos substituintes acil e amino sobre a ligação dupla (o que também é denominado efeito "pushpull" de elétrons) $)^{1}$. A deslocalização de elétrons $\pi$, decorrente deste efeito, tem como consequiência uma notável polarização destes carbonos olefínicos (como se pode constatar pelos seus deslocamentos químicos de ${ }^{13} \mathrm{C}$ não usuais $)^{2}$, conferindo a estes compostos uma natureza reativa distinta de seus correlatos estruturais enamina e enona (pela estabilização de seus estados de transição polares) ${ }^{3}$.

O efeito mesomérico em enaminonas também é responsável pela coexistência de isômeros conformacionais e configuracionais, devido à restrição rotacional de ligações $\sigma$ (aumento do caráter $\pi$ das ligações N-C e C-CO) e à atenuação da barreira de isomerização da dupla ligação olefínica (caráter $\pi$ diminuído) ${ }^{4}$, esta última podendo ocorrer até mesmo à temperatura ambiente 5 . Quatro formas isoméricas (Esquema 1) são possíveis, e a predominância e a interconversão entre elas dependem de diversos fatores estruturais. $\mathrm{O}$ tipo e o volume de substituintes em enaminonas acíclicas podem favorecer uma determinada forma isomérica, em razão de uma menor tensão estrutural ou de uma maior planaridade para conjugação de elétrons $\pi^{6,7}$. $\mathrm{O}$ tautomerismo imino-enamina também pode favorecer o equilíbrio conformacional $^{8}$, e o isômero cis-s-cis pode ser estabilizado por ponte de hidrogênio intramolecular $\left(\mathrm{R}^{1}=\mathrm{H}\right)^{9-11}$. A isomeria conformacional também é observada para o grupo amino $\left(\mathrm{R}^{1} \neq \mathrm{R}^{2}\right)$, cuja restrição rotacional se intensifica proporcionalmente ao grau de conjugação no sistema enaminona ${ }^{12}$ (neste caso a nomenclatura pode ser do tipo $E-s-E-S-E)^{13}$.

\footnotetext{
*e-mail: hmferraz@iq.usp.br
}

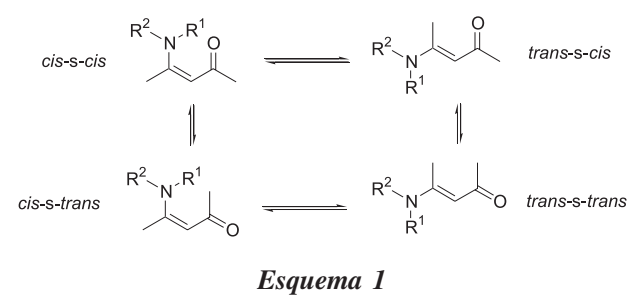

O grau de deslocalização de elétrons $\pi$ também varia para cada forma isomérica e responde por suas diferentes propriedades físicas, químicas e espectroscópicas. Os deslocamentos químicos de ${ }^{13} \mathrm{C}$ e ${ }^{15} \mathrm{~N}$ em enaminonas ${ }^{14-16}$ refletem esta tendência, variando em função do padrão estrutural da enaminona (acíclica, carbocíclica ou heterocíclica) e de sua porção amino (acíclica, cíclica ou aromática). Sete possíveis padrões estruturais (A-G) são descritos na Figura 1 para as enaminonas.

Da mesma forma, o padrão estrutural também pode determinar a reatividade do sistema enaminona $\left(\mathrm{N}_{a}-\mathrm{C}_{b}=\mathrm{C}_{c}-\mathrm{C}_{d}=\mathrm{O}_{e}\right)$, através da distribuição eletrônica ao longo de seus centros reativos $(a-c-e$, nucleofílicos, e $b$ - $d$, eletrofílicos) e da distância interatômica entre eles. A resistência à redução da ligação dupla nas enaminonas do tipo D1 e D2 $(\mathrm{n}=1)$ por $\mathrm{NaBH}(\mathrm{OAc}){ }_{3}^{17}$ ou $\mathrm{NaBH}_{3} \mathrm{CN}^{18}$ deve-se à forma isomérica trans-s-trans (restrita), onde a distância interatômica $\mathrm{N}_{\mathrm{a}}-\mathrm{O}_{\mathrm{e}}$ impossibilita a coordenação destes com o átomo de boro. Já em heterociclos nitrogenados do tipo $\mathbf{E} 2^{17}$ e $\mathbf{F} 2^{19}$ a coordenação entre estes átomos é possível, viabilizando a redução com estes reagentes.

$\mathrm{O}$ padrão de substituição nas enaminonas também altera a reatividade destes centros. Assim, o centro $\mathrm{N}_{a}$ de uma $\beta$-enamino cetona deve ser menos nucleofílico que o de um $\beta$-enamino éster, devido ao efeito retirador de elétrons mais acentuado da função cetona $^{20}$

Devido à sua estrutura bidentada e insaturada, enaminonas são capazes de formar quelatos estáveis com $\mathrm{Cu}$ (II), $\mathrm{Ni}$ (II) e $\mathrm{VO}(\mathrm{II})^{21}$. A estrutura cristal-líquida ${ }^{22} \mathrm{e}$ as propriedades magnéticas ${ }^{23}$ e catalíticas ${ }^{24}$ destes complexos, bem como a atividade anti-convulsivante de enaminonas ${ }^{25,26}$, têm sido estudadas à luz destas interações ${ }^{27}$, em conjunto com o perfil espectroscópico destes compostos ${ }^{2,28,29}$.

Duas revisões sobre enaminonas ${ }^{30,31}$ e outras duas sobre enaminas, abrangendo também as enaminonas ${ }^{32,33}$, cobrem a literatura até 1993. 
$R^{2} R^{1} O$

A1

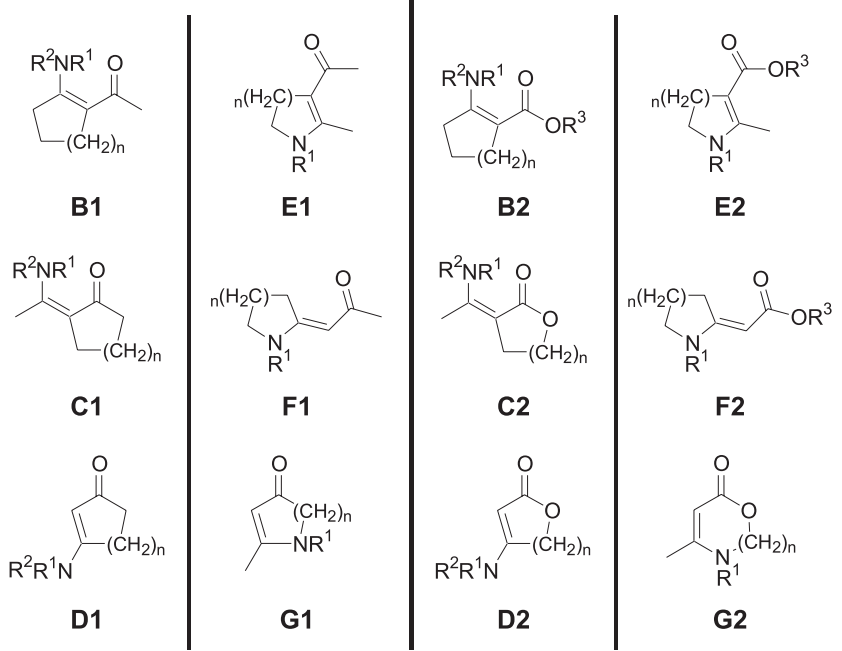

Figura 1. Padrões Estruturais de Enaminonas

Uma revisão mais recente sobre pirrróis cita a preparação destes a partir de algumas enaminonas ${ }^{34}$.

\section{SÍNTESES}

\section{Condensação de compostos $\beta$-dicarbonílicos com aminas}

O método mais comum de preparação de enaminonas é a condensação de compostos $\beta$-dicarbonílicos com aminas primárias ou secundárias, em refluxo de benzeno ou tolueno, com remoção azeotrópica de água ${ }^{35}$. Adaptações bastante comuns desta reação envolvem o uso alternativo de acetatos de aminas voláteis ou amônia ${ }^{36}$, o emprego de catalisadores ácidos, como o ácido ascórbico ${ }^{37}$, e a utilização de derivados carbonílicos mais reativos, como ésteres ou cloretos de ácido vinílogos (no caso de aminas pouco básicas) ${ }^{38}$, usando acetonitrila como solvente, quando a amina estiver na forma de cloridrato ${ }^{39}$.

A condensação de aminas com derivados 1,3-dicarbonílicos, em refluxo de benzeno ou tolueno, ainda hoje é utilizada na preparação de enaminonas (eq. 1 no Esquema 2) ${ }^{40}$. Adaptações recentes deste método (Esquema 2) envolvem o uso de peneira molecular $4 \AA$ (eq. 2 e 3$)^{41,42}$ e de catalisadores como $\mathrm{BF}_{3} \cdot \mathrm{Et}_{2} \mathrm{O}$ (eq. 3) $)^{42}$ TsOH (eq. 4) ${ }^{43-46}$ e TMSTf (eq. 5$)^{47}$. Enamido ésteres também podem ser obtidos por condensação em benzeno / $\mathrm{TsOH}^{46}$.

A condensação de aminas com compostos $\beta$-dicarbonílicos tem sido realizada também em outras condições (Esquema 3), como $\mathrm{Et}_{2} \mathrm{O}$ em presença de $\mathrm{TiCl}_{4}$ (eq. 6) ${ }^{48}$, DMF em presença de TMOF (eq. $7)^{49,50}$, THF (eq. 8$)^{51}, \mathrm{MeOH}$ (eq. 8 e 9) $)^{51,52}, \mathrm{MeCN}$ (eq. 10) ${ }^{13}$ e até mesmo $\mathrm{H}_{2} \mathrm{O}$ (eq. 11 e 12$)^{53}$ e catalisador de ouro(III) ${ }^{54}$.

Enaminonas também têm sido preparadas na ausência de solvente (Esquema 4), pela simples mistura dos reagentes (reação em estado sólido, eq. 13$)^{55}$, ou em suporte sólido $\left(\mathrm{Al}_{2} \mathrm{O}_{3}{ }^{56,57}\right.$, eq. 14 , ou Montmorillonita $\mathrm{K}-10^{58}$, eq. 15) ou em tubo selado (eq. 16$)^{28}$.

\section{Adição de aminas a compostos carbonílicos $\alpha, \beta$-insaturados}

Reações do tipo adição de Michael podem ocorrer entre aminas e compostos acetileno-, aleno- ou etileno-carbonílicos. Tanto a adição à ligação tripla como ao aleno fornecem diretamente a enaminona.
No caso de enonas, a ligação $\mathrm{C}=\mathrm{C}$ deve ser regenerada após a adição da amina.

Métodos citados em revisões anteriores englobam a adição de aminas a acetileno-ésteres ou cetonas ${ }^{59-61}$ e a trimetil silil etinil cetonas $^{62}$. A adição de trifenilsilil acetileto de lítio a amidas leva à formação de enaminonas, por subseqüiente adição do amideto de lítio à acetilenocetona, ambos formados na reação ${ }^{63}$. A adição de arilaminas a enonas não-funcionalizadas produz enaminonas em rendimentos moderados, quando catalisada por $\mathrm{PdCl}_{2}\left(\mathrm{CH}_{3} \mathrm{CN}\right.$ ) (na presença de benzoquinona e cloreto de lítio) ${ }^{64,65}$. Quando adequadamente funcionalizadas, enonas podem reagir com aminas pouco básicas

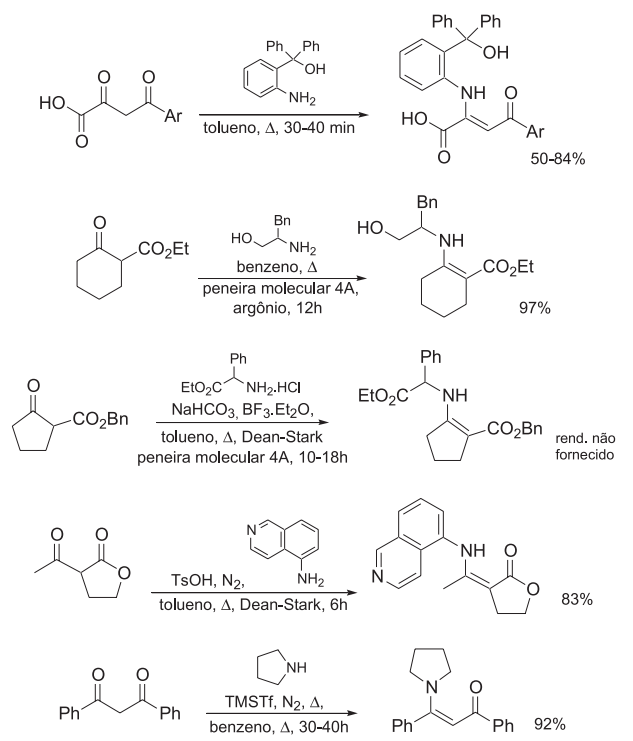

Esquema 2

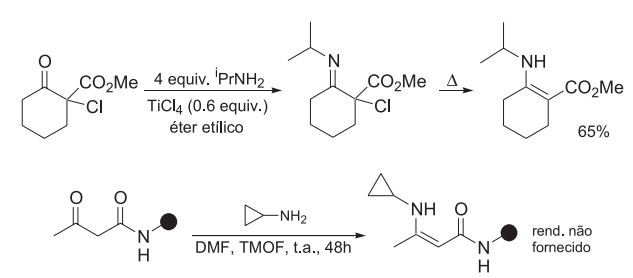

(eq. 6)

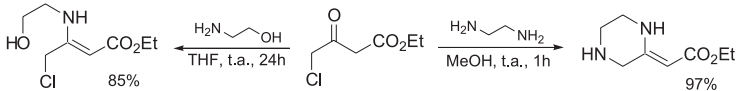

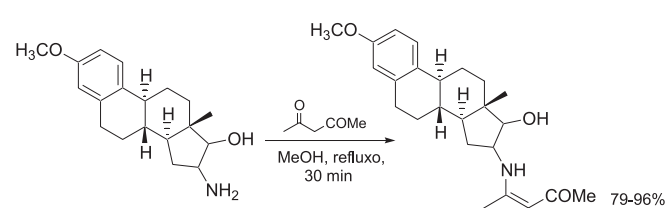

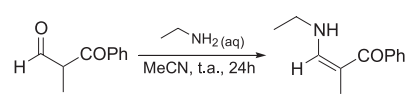

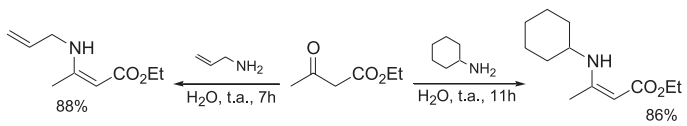

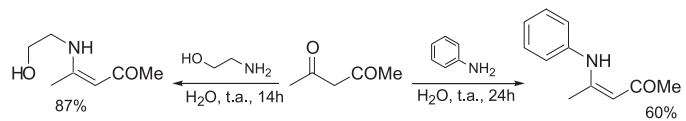



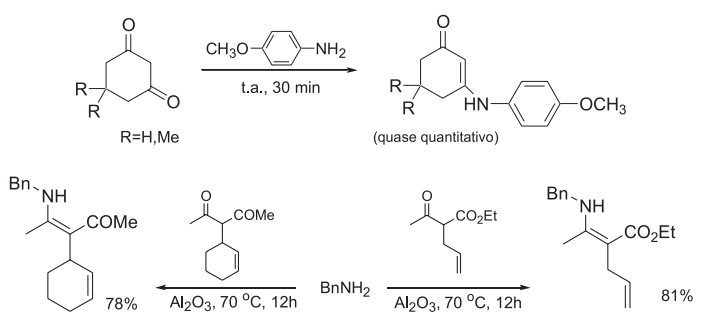

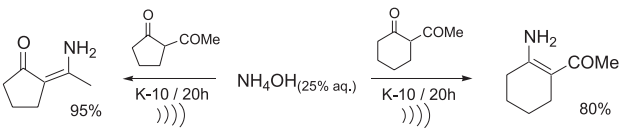

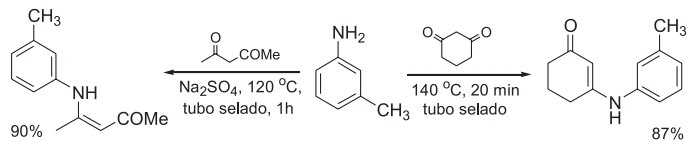

Esquema 4

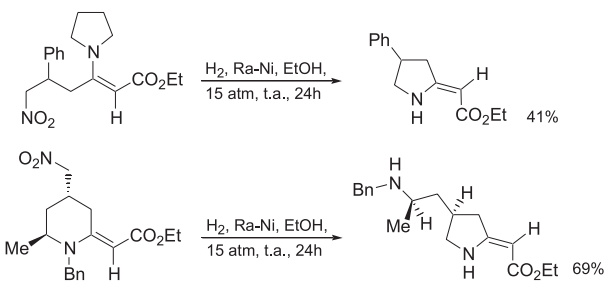

Esquema 9

\section{Esquema 5}

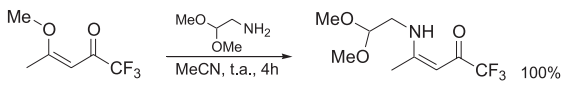

Esquema 6

Iodo-acrilatos podem gerar enaminonas por reação com aminas primárias e secundárias, em ótimos rendimentos ${ }^{69}$. Enonas sulfonil ${ }^{70}$ (Esquema 7) ou triclorometil ${ }^{71}$ (Esquema 8) substituídas surgem como substratos inusitados na preparação de enaminonas.

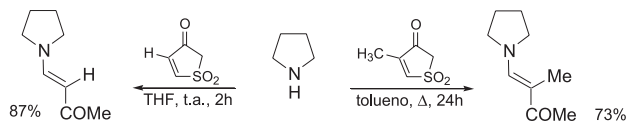

Esquema 7

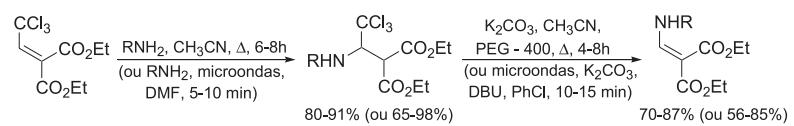

Esquema 8

Outro caso interessante se refere à transaminação intramolecular de enamino ésteres ${ }^{72}$, onde o grupo abandonador pode ficar retido ou não no produto, dependendo da estrutura do substrato (Esquema 9).
A adição de anilina a um derivado glicosídico da 4-hidróxibutenolida ocorre em seu carbono $\alpha$-carbonílico. A enaminona é formada pela hidrólise do pseudo-cetal, com subsequiente eliminação de ácido fórmico ${ }^{73}$ (Esquema 10).

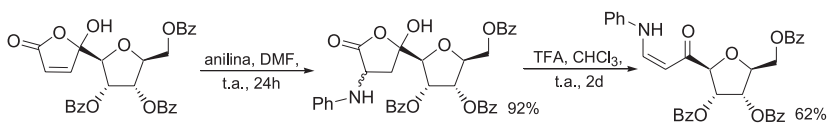

Esquema 10

Enaminonas podem ser preparadas a partir de aril enonas não funcionalizadas de duas formas distintas (Esquema 11): a) pela adição de metoxiamina a uma chalcona ${ }^{74}$ (eq. 19), seguida de eliminação de $\mathrm{MeOH}$ catalisada por base; b) pela adição de anilina ao ácido cinâmico $^{75}$ (eq. 20), mediada por triacetato de tálio, em dioxano. Esta reação representa uma variante não catalítica do uso de $\mathrm{PdCl}_{2}\left(\mathrm{CH}_{3} \mathrm{CN}\right)_{2}$.

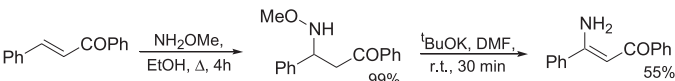

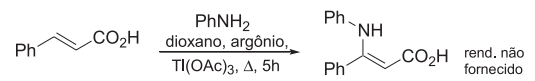

\section{Esquema 11}

\section{Condensação entre compostos carbonílicos e compostos aza- insaturados}

A construção de enaminonas pode ser efetuada pela condensação entre sistemas carbonílicos (aldeídos, cetonas ou derivados de ácido) e compostos aza-insaturados (nitrilas, iminas ou derivados de imidas), pela formação de uma ligação C-C. Um destes compostos deverá conter hidrogênios ácidos, para formar um carbânion que atue como nucleófilo no ataque ao outro composto (exceto na reação de Blaise). Dependendo da natureza deste último, a reação poderá ser de adição (no caso de nitrilas) ou de substituição nucleofílica a carbono trigonal.

\section{Adição de ésteres a nitrilas (reação de Blaise)}

Enamino ésteres podem ser preparados pela reação entre nitrilas e $\alpha$-bromo ésteres, na presença de zinco. Os baixos rendimentos da reação de Blaise ${ }^{76}$ são devidos a reações paralelas e, portanto, sua amplitude limitada obscureceu este método por mais de 80 anos. Quando a reação foi finalmente reestudada e viabilizada por ligeiras modificações no protocolo, forneceu enamino ésteres em ótimos rendimentos ${ }^{77}$. A adição a nitrilas também pode ser efetuada por uso do enolato de magnésio derivado de ésteres, após tentativas infrutíferas de adição via enolatos de lítio ${ }^{78}$. 
Uma versão mais recente da reação de Blaise envolve a sonicação dos reagentes com zinco metálico e subseqüente tratamento com $\mathrm{K}_{2} \mathrm{CO}_{3 \text { (aq) }}$, obtendo-se os produtos com rendimentos variando de médios a bons ${ }^{79}$ (Esquema 12).

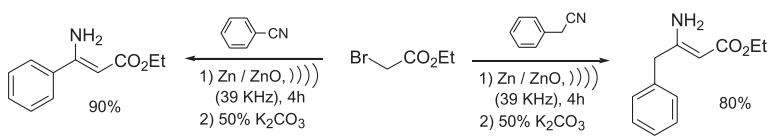

Esquema 12

Reações de substituição nucleofílica em carbono trigonal

A substituição nucleofílica em carbono trigonal pode gerar enaminonas por três tipos de reação, dependendo dos reagentes: a) acetilação de enaminas; b) acetilação de aza-enolatos e c) imidoilação de enolatos.

a) Acetilação de enaminas - Enaminas terciárias podem atuar como nucleófilos frente a cloretos de acila (na presença de $\left.\mathrm{Et}_{3} \mathrm{~N}\right)^{80}$ ou ésteres (por condensação intramolecular) ${ }^{81}$, formando enamino cetonas. Um método mais recente descreve a formação de $\beta$-enamino$\alpha$ '-trifluorocetonas pelo uso de um trifluoracetato de piridínio ${ }^{82}$ (Esquema 13). Iminas também foram acetiladas por fluoretos de perfluoroalcanoíla $^{83}$ ou $N, N$-carbonildiimidazo ${ }^{84}$.
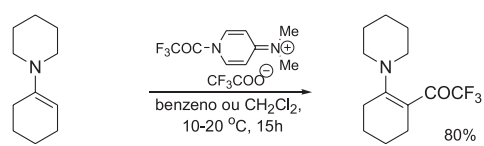

Esquema 13

b) Acetilação de aza-enolatos - Trata-se de uma reação que vem ganhando destaque ao longo dos anos. Iminas secundárias podem formar aza-enolatos de lítio por tratamento com n-BuLi ${ }^{85,86}$ ou $\mathrm{LDA}^{87,88}$, efetuando substituição nucleofílica em ésteres ${ }^{85-89}$, cloretos de ácido ${ }^{85}$ e na acetilacetona ${ }^{85}$ (por deslocamento de acetona). Outros substratos como benzotriazolilcetonas ${ }^{90}$, ésteres aromáticos ${ }^{91} \mathrm{e}$ $\alpha$-fluoroésteres ${ }^{92}$ (Esquema 14), além de carbonatos e cloroformiatos $^{93}$, têm sido utilizados nos últimos anos.
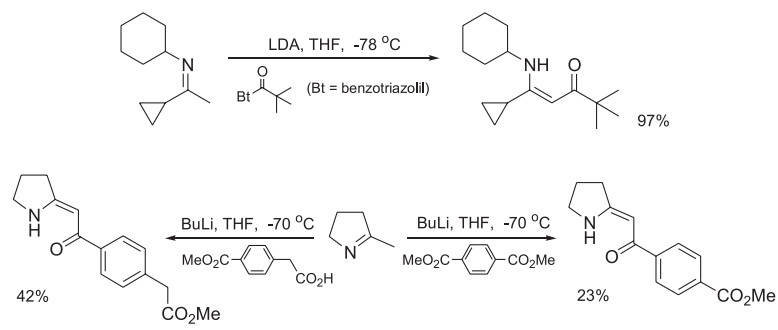

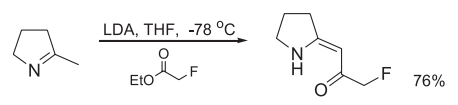

Esquema 14

c) Imidoilação de enolatos - Esta reação foi, inicialmente, efetuada entre o enolato de lítio do acetato de metila e uma tosil imina ${ }^{94}$. Cloretos de imidoíla têm sido utilizados na preparação de $\beta$-enamino cetonas $^{92}$ e $\beta$-enamino ésteres ${ }^{95}$ (Esquema 15 ).

Derivados heterocíclicos de $\beta$-enaminonas também podem ser obtidos por substituição nucleofílica em ésteres ${ }^{96}$ ou cloretos de
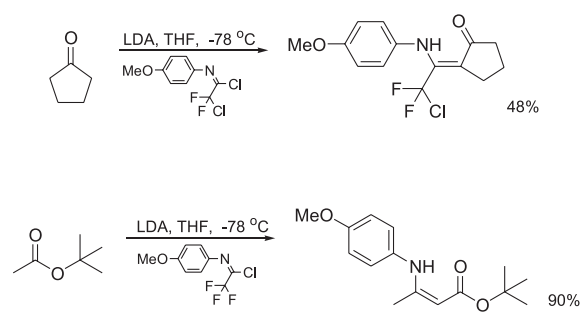

Esquema 15

imidoíla ${ }^{97,98}$ (eq. 21 e 22, Esquema 16) e também por adição de azaenolatos a nitrilas ${ }^{99}$ (Esquema 17).
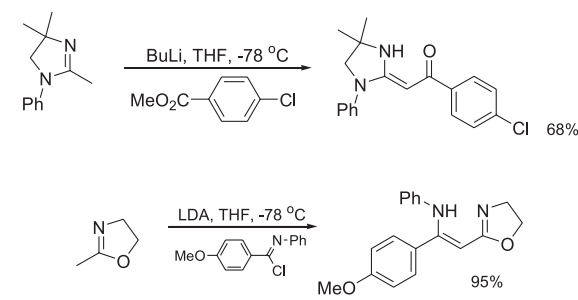

(eq. 22)

Esquema 16

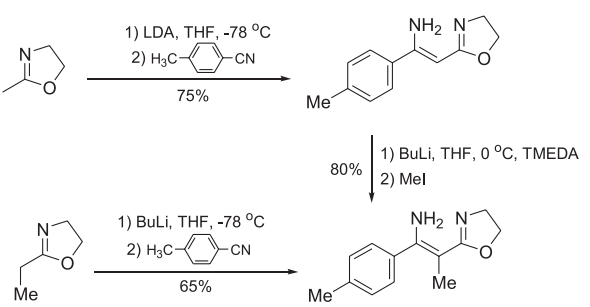

Esquema 17

Uma variação deste tipo de reação ("one-pot”) produz a enaminona em rendimentos que variam de bons a ótimos ${ }^{97,99}$ (Esquema 18).

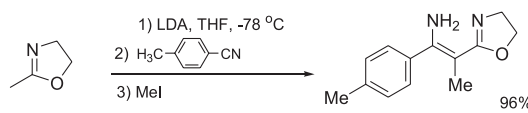

Esquema 18

\section{OUTROS MÉTODOS}

Sintese de Eschenmoser

Este tipo de reação é aplicado comumente na síntese de enaminonas do tipo F1 e F2 (Figura 1). O intermediário chave é um sal de imínio cíclico, cuja hidrólise leva à formação de um tioimino éster, que por tratamento com trifenilfosfina sofre extrusão de enxofre, dando origem à enaminona ${ }^{100}$. $\mathrm{O}$ sal de imínio também pode reagir com compostos que possuem metilenos ácidos, formando diretamente a enaminona ${ }^{101}$. Os compostos metileno ácidos, por sua vez, também podem formar enaminonas a partir de éteres lactímicos ${ }^{102}$, em uma versão do método. Uma outra modificação apresenta uma versão "one-pot" da extrusão de enxofre ${ }^{103}$ (Esquema 19). 


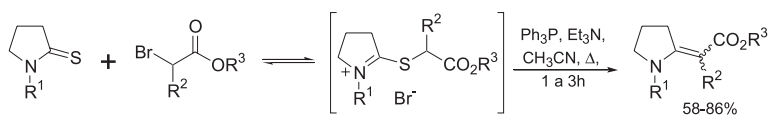

Esquema 19

Reações de Mannich Modificadas

A reação de Mannich envolve a condensação de compostos contendo um grupo ácido com formaldeído e uma amina, na presença de um ácido forte, para formar uma base de Mannich ${ }^{104}$. Uma série de versões desta famosa reação é descrita na literatura para a preparação de enaminonas ${ }^{105}$. As reações de cetonas com acetais de amidas ${ }^{106,107}$ ou com trietilortoformiato na presença de uma amina secundária $^{108}$ são alguns exemplos recentes (Esquema 20).

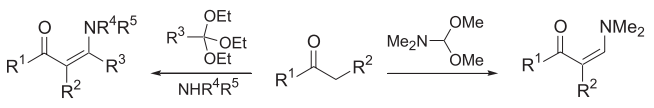

\section{Esquema 20}

\section{Abertura de isoxazóis}

A abertura de anéis isoxazolínicos por meio de redução da ligação N-O, comumente por hidrogenação com níquel de Raney ${ }^{109}$, dá origem a enaminonas primárias. Mais recentemente, a redução com Ni-Raney foi aplicada a diidro e tetraidroisoxazóis, dando origem a enaminonas ${ }^{110}$. Um método para isomerização de aril-enamino cetonas via isoxazol ${ }^{111}$ faz uso de hexacarbonila de molibdênio em água/ acetonitrila para a abertura do ane ${ }^{112}$. A redução também pode ser feita com $\mathrm{SmI}_{2}{ }^{113}$ ou por hidrogenação com $\mathrm{Pd} / \mathrm{C}^{114}$ (Esquema 21).

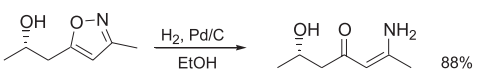

Esquema 21

\section{Decomposição de enaminonas derivadas do ácido de Meldrum}

A obtenção de enaminonas por este tipo de reação é uma conseqüência da formação, por pirólise, de enamino-derivados do ácido de Meldrum, através de um intermediário bastante reativo, o aminometilenoceteno. Este intermediário, em altas temperaturas $\left(800{ }^{\circ} \mathrm{C}\right)$, dá origem a uma estrutura cíclica do tipo $\mathbf{G 1}$ (Figura $1, \mathrm{n}=1$ ) $^{115}$, ou a um $\beta$-enamino ácido ou éster, por tratamento com água ou álcool ${ }^{116}$. A estrutura do substituinte ligado ao nitrogênio pode determinar o rumo da reação (Esquema 22). Assim, derivados N-cloro-alquilados levam à formação de enaminonas do tipo E2 (eq. 23), enquanto que
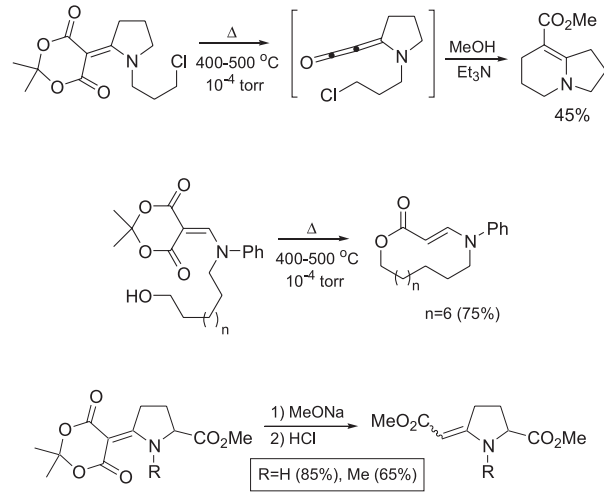

derivados N- hidróxi-alquilados formam enaminonas do tipo $\mathbf{G 2}$ (eq. 24). No primeiro caso, forma-se um enamino éster aza-bicíclico ${ }^{117}$; no segundo caso, forma-se uma $\beta$-enamino aza-lactona macrocíclica ${ }^{118}$. Este método também foi aplicado na síntese de 5carbóxi-metileno-2-carbóxi-pirrolidino derivados do tipo F2 (eq. 25) 119,120 .

\section{Outras reações}

Enaminonas heterocíclicas do tipo E2 podem ser obtidas pela adição de aminas a $\beta$-acetóxi- $\alpha$-metileno ésteres, derivados da reação de Baylis-Hillman, ${ }^{121}$ seguida de substituição nucleofílica aromática (Esquema 23) ${ }^{122}$. O produto inicialmente formado se isomeriza à enaminona desejada, quando mantido em solução de diclorometano por três dias.

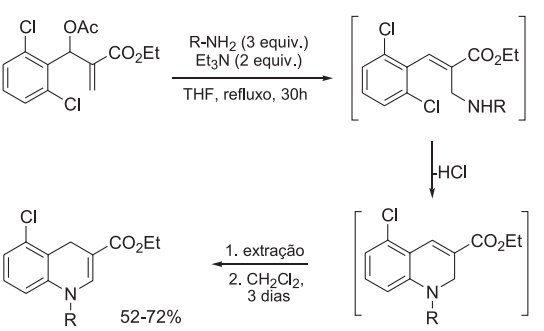

Esquema 23

$\mathrm{N}$-acil-enaminonas cíclicas do tipo $\mathbf{E}$ também podem ser produzidas por formilação ou acetilação de enamidas, nas condições das reações de Vilsmeier ou de Friedel-Crafts, respectivamente ${ }^{123}$ (Esquema 24, eq. 26). A cloroformilação de lactamas pela reação de Vilsmeier-Haack ${ }^{124}$ também é útil na síntese de enaminonas do tipo E (Esquema 24, eq. 27).

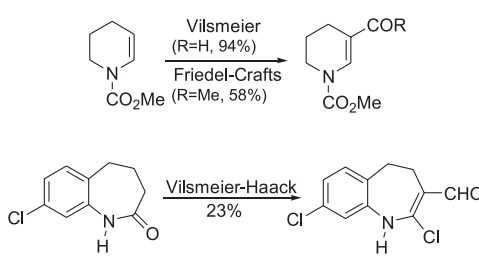

Esquema 24

Enaminonas tetraidroquinolínicas do tipo F1 ou F2 (Figura 1, $\mathrm{n}=2$ ) podem ser obtidas por ciclocondensação de ceteno tio-acetais induzida por $\mathrm{HgCl}_{2}$ ou $\mathrm{POCl}_{3}{ }^{125}$, como mostrado no Esquema 25 .

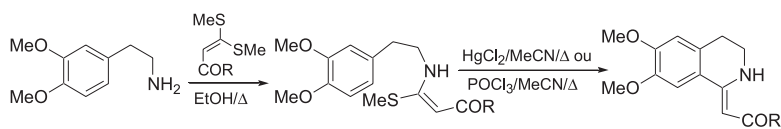

Esquema 25

Enaminonas diidropiridínicas do tipo $\mathbf{E 2}(\mathrm{n}=2)$ podem ser obtidas por tratamento de iminofosforil-enonas com aldeídos aromáti$\cos$ a $160{ }^{\circ} \mathrm{C}$ em uma reação do tipo aza-Wittig ${ }^{126}$. $\mathrm{O}$ mesmo produto pode ser obtido em condições mais brandas, pela conversão prévia do intermediário reativo em seu sal de enamino-fosfônio, por tratamento com $\mathrm{HCl}^{127}$ (Esquema 26).

A reação de imino-Diels-Alder entre iminas aromáticas e o dieno de Danishefsky leva à formação de diidropiridonas do tipo $\mathbf{1 g}(\mathrm{n}=2)$ 
A reação pode ser catalisada por iodeto de samário ${ }^{128}$ ou por Nafion ${ }^{129}$ em ácido trifluoroacético ${ }^{130}$ (Esquema 27).

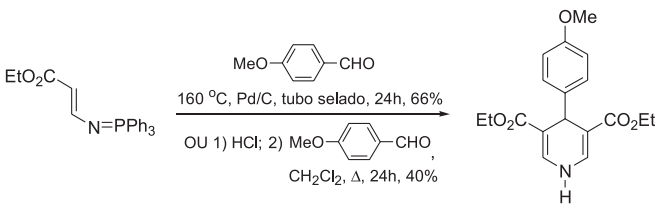

Esquema 26

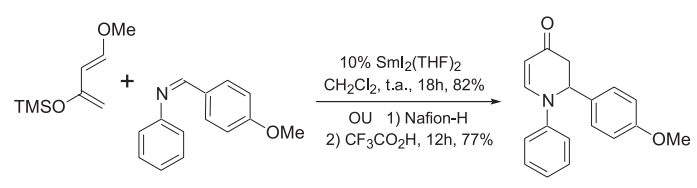

Esquema 27

\section{CONCLUSÃO}

Os artigos de revisão citados no início deste trabalho apresentam uma série de métodos para a construção de enaminonas. Foram destacados aqui aqueles que se mantêm mais utilizados (aperfeiçoados ou não), além de novos métodos encontrados na literatura a partir de 1993. No momento, estamos preparando um segundo artigo de revisão, que versará sobre a reatividade de enaminonas, com enfoque nas sínteses que envolvem enaminonas como materiais de partida ou como intermediários sintéticos.

\section{AGRADECIMENTOS}

\section{FAPESP, CNPq, CAPES.}

\section{REFERÊNCIAS}

1. Sandstrm, J.; Top. Stereochem. 1983, 14, 83.

2. Morales-Ríos, M. S.; Suárez-Castillo, O. R.; Alvarez-Cisneros, C.; JosephNathan, P.; Can. J. Chem. 1999, 77, 130.

3. Morales-Ríos, M. S.; García-Velgara, M.; Cuevas-Cervantes, H.; AlvarezCisneros, C.; Joseph-Nathan, P.; Magn. Reson. Chem. 2000, 38, 172.

4. Osman, R.; Shvo, Y.; Tetrahedron 1978, 34, 2321.

5. Fischer, G.; Rudorf, W.-D.; Kleinpeter, E.; Magn. Reson. Chem. 1991, 29, 212.

6. Dabrowski, J.; Kozerski, L.; Org. Magn. Reson. 1973, 5, 469.

7. Filleux-Blanchard, M.-L.; Durand, H.; Martin, G. J.; Org. Magn. Reson. 1970, 2, 539.

8. Katritzky, A. R., Ghiviriga, I., Oniciu, D. C.; O’Ferrall, R. A. M., Walsh, S. M.; J. Chem. Soc., Perkin Trans. 2 1997, 2605.

9. Gómez-Sánchez, A.; Valle, A. M.; J. Chem. Soc., Perkin Trans. 1 1973, 15.

10. Gilli, P.; Bertolasi, V.; Ferreti, V.; Gilli, G.; J. Am. Chem. Soc. 2000, 122, 10417.

11. Bertolasi, V.; Gilli, P.; Ferreti, V.; Gilli, G.; Acta Crystallogr., Sect. B: Struct. Sci. 1998, B54, 50

12. Filleux-Blanchard, M.-L.; Durand, H.; Martin, G. J.; Org. Magn. Reson. 1970, 2, 539 .

13. Zhuo, J.-C.; Mag. Res. Chem. 1998, 36, 565.

14. Ahmed, M. G.; Hickmott, P. W.; J. Chem. Soc., Perkin Trans. 2 1977, 838.

15. Schwotzer, W.; Philipsborn, W. von; Helv. Chim. Acta 1977, 60, 1501.

16. Westerman, P. W.; Roberts, J. D.; J. Org. Chem. 1977, 42, 2249.

17. Cimarelli, C.; Palmieri, G.; J. Org. Chem. 1996, 61, 5557;.

18. Ramesh, N. G.; Klunder, A. J. H.; Zwanenburg, B.; Tetrahedron Lett. 1998, 39, 1429.

19. Nikiforov, T.; Stanchev, S.; Milenkov, B.; Dimitrov, V.; Heterocycles 1986, 24, 1825.

20. Pereira, F. L. C.; Ferraz, H. M. C.; resultados não publicados.

21. Szydlowska, J.; Krówczynski, A.; Górecka, E.; Pociecha, D.; Inorg. Chem. 2000, 39, 4879 .
22. Krówczynski, A.; Szydlowska, J.; Górecka, E.; Liq. Cryst. 1999, 26, 685.

23. Musin, R. N.; Ovcharenko I. V.; Ohrström, L.; Rey, P.; J. Struct. Chem. 1997, 38, 703 .

24. Zakharov, A. N.; Zefirov, N. S.; Kinetics Catal. 1998, 39, 33.

25. Cox, D. S.; Du, J.; Scott, K. R., Gao, H.; Eddington, N. D.; J. Chromatogr. B: Biomed. Sci. Appl. 2000, 749, 191.

26. Foster, J. E.; Nicholson, J. M.; Butcher, M.; Stables, P. T.; Edafiogho, I. O.; Goodwin, A. M.; Henson, M. C.; Smith, A. C.; Scott, K. R.; Bioorg. Med. Chem. 1999, 7, 2415.

27. Kubicki, M.; Bassyouni, H. A. R.; Codding, P. W.; J. Mol. Struct. 2000, $525,141$.

28. Zhuo, J.-C.; Mag. Res. Chem. 1997, 35, 311.

29. Gómez-Sánchez, A.; Paredes-León, R.; Cámpora, J.; Magn. Reson. Chem. 1998, 36, 154.

30. Greenhill, J. V.; Chem. Soc. Rev. 1977, 6, 277.

31. Lue, P.; Greenhill, J. V. Adv. Heterocycl. Chem. 1997, 67, 207.

32. Granik, V. G.; Russ. Chem. Rev. (Engl. Transl.) 1984, 53, 383.

33. Cervinka, O. In The Chemistry of Enamines; Rapoport, Z., Ed.; Wiley: Chichester,1994, p.467.

34. Ferreira, V. F.; de Souza, M. C. B. V.; Cunha, A. C.; Pereira, L. O. R.; Ferreira, M. L. G.; Org. Prep. Proced. Int. 2001, 33, 411.

35. Dixon, K.; Greenhill, J. V.; J. Chem. Soc., Perkin Trans.1 1974, 164

36. Baraldi, P. G.; Simoni, D.; Manfredini, S.; Synthesis 1983, 902.

37. Okamoto, T.; Shudo, K.; Tetrahedron Lett. 1973, 4533.

38. Dixon, K.; Greenhill, J.V.; J. Chem. Soc., Perkin Trans.1 1976, 2211.

39. Hojo, M.; Massuda, R.; Okada, E.; Sakaguchi, S.; Narumiya, H.; Morimoto, K.; Tetrahedron Lett. 1989, 30, 6173.

40. Kolotova, N. V.; Koz'minykh, V. O.; Dolbilkina, E. V.; Koz'minykh, E. N.; Russ. Chem. Bull. 1998, 47, 2246.

41. Aoyagi, Y.; Mizusaki, T.; Ohta, A.; Tetrahedron Lett. 1996, 37, 9203.

42. Benovsky, P.; Stephenson, G. A.; Stille, J. R.; J. Am. Chem. Soc. 1998, 120, 2493.

43. Parr, R. W.; Reiss, J.A.; Aust. J. Chem. 1984, 37, 389.

44. Yapi, A. D.; Mustofa, M.; Valentin, A.; Chavignon, O.; Teulade, J.-C.; Mallie, M.; Chapat, J.-P.; Blache, Y.; Chem. Pharm. Bull. 2000, 48, 1886.

45. Fukuda, Y.; Furuta, H.; Shiga, F.; Asahina, Y.; Terashima, S.; Heterocycles 1997, 45, 2303.

46. Ovenden, S. P. B.; Capon, R. J.; Lacey, E.; Gill, J. H.; Friedel, T.; Wadsworth, D.; J. Org. Chem. 1999, 64, 1140.

47. Cartaya-Marin, C. P.; Henderson, D. G.; Soeder, R. W.; Zapata, A. J.; Synth. Commun. 1997, 27, 4275.

48. Stevens, C. V; Kesteleyn, B.; Alonso, E. R.; De Kimpe, N.; Tetrahedron 2001, 57, 7685 .

49. Trautwein, A. W.; Süßmuth, R. D.; Jung, G.; Bioorg. Med. Chem. Lett. 1998, 8,2381

50. Trautwein, A. W.; Jung, G.; Tetrahedron Lett. 1998, 39, 8263.

51. Puebla, P.; Honores, Z.; Medarde, M.; Caballero, E.; San Feliciano, A.; Morán, L.; J. Heterocycl. Chem. 1999, 36, 1097.

52. Dubs, M.; Krieg, R.; Görls, H.; Schönecker, B.; Steroids 2000, 65, 305

53. Stefani, H. A.; Costa, I. M.; Silva, D. O.; Synthesis 2000, 1526.

54. Arcadi, A.; Bianchi, G.; Di Giuseppe, S.; Marinelli, F.; Green Chem. 2003, 5,64 .

55. Kaupp, G.; Schmeyers, J.; Boy, J.; J. Prakt. Chem. 2000, 342, 269.

56. Ferraz, H. M. C.; Pereira, F. L. C.; Leite, F. S.; Nunes, M. R. S.; PayretArrúa, M. E.; Tetrahedron 1999, 55, 10915.

57. Ferraz, H. M. C.; de Oliveira, E. O.; Payret-Arrúa, M. E.; Brandt, C. A.; J. Org. Chem. 1995, 60, 7357.

58. Valduga, C. J.; Squizani, A.; Braibante, H. S.; Braibante, M. E. F.; Synthesis 1998, 1019.

59. Bowden, K.; Braude, E. A.; Jones, E. R. H.; Weedon, B. C. L.; J. Chem. Soc. 1946, 45.

60. Winterfeldt, E.; Welke, J. M.; Chem. Ber. 1968, 101, 2381.

61. Linderman, R. J.; Kirollos, K. S.; Tetrahedron Lett. 1990, 31, 2689.

62. Wilbur Jr., J. M.; Wilbur, E. D.; Macromolecules 1990, 23, 1894.

63. Suzuki, K.; Ohkuma, T.; Tsuchihashi, G.-I.; J. Org. Chem. 1987, 52, 2929.

64. Bozell, J. J.; Hegedus, L. S.; J. Org. Chem. 1981, 46, 2561.

65. Murahashi, S.-I.; Mitsue, Y.; Tsumiyama, T.; Bull. Chem. Soc. Jpn. 1987, 60,3285

66. Alberola, A.; Andrés, J. M.; González, A.; Pedrosa, R.; Vicente, M.; Heterocycles 1990, 31, 1049.

67. Jie, M. S. F. L. K.; Lau, M. M. L.; Lipids 2000, 35, 1135.

68. Okada, E.; Masuda, R.; Hojo, M.; Yoshida, R.; Heterocycles 1992, 34, 1435.

69. Maw, G.; Thirsk, C.; Whiting, A.; Tetrahedron Lett. 2001, 42, 8387.

70. Hofslokken, N.U.; Skattebol, L.; J. Chem. Soc., Perkin Trans. 1 1999, 3085.

71. Deshmukh, A. R. A. S.; Panse, D. G.; Bhawal, B. M.; Synth. Commun. 1999, 29, 1801.

72. Sosnicki, J. G.; Liebscher, J.; Synlett 1996, 1117. 
73. Maeba, I.; Ito, Y.; Wakimura, M.; Ito, C.; Heterocycles 1993, 36, 2805.

74. Seko, S.; Tani, N.; Tetrahedron Lett. 1998, 39, 8117.

75. Fukushima, M.; Tatsumi, K.; Morimoto, K.; Environ. Sci. Technol. 2000, 34, 2006.

76. Blaise, E. E.; C. R. Hebd. Seances Acad. Sci. 1901, 132, 478.

77. Hannick, S. M.; Kishi, Y.; J. Org. Chem. 1983, 48, 3833.

78. Hiyama, T.; Kobayashi, K.; Tetrahedron Lett. 1982, 23, 1597.

79. Lee, A. S.-Y.; Cheng, R.-Y.; Tetrahedron Lett. 1996, 38, 443.

80. Hünich, S.; Benzing, E.; Lücke, E.; Chem. Ber. 1957, 90, 2833.

81. Meyers, A. I.; Reine, A. H.; Sincar, J. C.; Rao, K. B.; Singh, S.; Weidmann, H.; Fitzpatrick, M.; J. Heterocycl. Chem. 1968, 5, 151.

82. Simchem, G.; Schimidt, A.; Synthesis 1997, 117.

83. Przyborowsli, J.; Lork, E.; Röschenthaler, G.-V.; J. Fluorine Chem. 2000, 104, 207.

84. Fustero, S.; de la Torre, M. G.; Jofré, V.; Carlón, R. P.; Navarro, A.; Fuentes, A. S.; J. Org. Chem. 1998, 63, 8836.

85. Dannhardt, G.; Obergrusberger, R.; Arch. Pharm. 1979, 312, 499.

86. Dannhardt, G.; Geyer, Y.; Obergrusberger, R.; Ziereis, K.; Chem. Zeit. 1987, $111,237$.

87. Bartoli, G.; Cimarelli, C.; Palmieri, G.; Bosco, M.; Dalpozzo, R.; Synthesis 1990, 895 .

88. Katritzky, A. R.; Barcock, R. A.; Long, Q.-H.; Balasubramanian, M.; Malhotra, M.; Greenhill, J. V.; Synthesis 1993, 233.

89. Wisniewski Jr., A.; Oliveira, A. R. M.; da Cunha, C. J.; Simonelli, F.; Marques, F. A.; J. Braz. Chem. Soc. 1999, 10, 369.

90. Katritzky, A. R.; Fang, Y.; Donkor, A.; Xu, J.; Synthesis 2000, 2019.

91. Danhardt, G.; Bauer, A.; Nowe, U.; J. Prakt. Chem. 1998, 340, 256.

92. Fustero, S.; de la Torre, M. G.; Pina, B.; Simón-Fuentes, A.; J. Org. Chem. 1999, 64, 5551.

93. Bartoli, G.; Cimarelli, C.; Dalpozzo, R.; Palmieri, G.; Tetrahedron 1995 $51,8613$.

94. Fukuyama, T.; Yung, Y. M.; Tetrahedron Lett. 1981, 22, 3759.

95. Fustero, S.; Pina, B.; Simón-Fuentes, A.; Tetrahedron Lett. 1997, 38, 6771.

96. Dannhardt, G.; Laufer, S.; Synthesis 1989, 12.

97. Fustero, S.; Navarro, A.; Díaz, D.; de la Torre, M. G.; Asensio, A.; Sanz, F.; Gonzáles, M. L.; J. Org. Chem. 1996, 61, 8849.

98. Liu-Gonzalez, M.; Sanz-Ruiz, F.; Navarro-Martinez, A.; de la Torre, M. G.; Fustero-Lardies, S.; Acta Crystallogr. Sect. C: Cryst. Struct. Commun. 1998, 54, 875.

99. Fustero, S.; Díaz, M. D.; Asensio, A.; Navarro, A.; Kong, J. S.; Aguilar, E.; Tetrahedron 1999, 55, 2695.

100. Roth, M.; Dobs, P.; Götschi, E.; Eschenmoser, A.; Helv. Chim. Acta 1971, 54,710 .
101. Gugelchuk, M. M.; Hart, D. J.; Tsai, Y.-M.; J. Org. Chem. 1981, 46, 3671. 102. Célérier, J.-P.; Deloisy, E.; Lhommet, G.; Maitte, P.; J. Org. Chem. 1979, 44, 3089.

103. Marchand, P.; Fargeau-Bellassoued, M.-C.; Bellec, C.; Lhommet, G.; Synthesis 1994, 1118.

104. Holy, N.; Fowler, R.; Burnett, E; Lorenz, R.; Tetrahedron 1979, 35, 613.

105. Abdulla, R. F.; Brinkmeyer, R. S.; Tetrahedron 1979, 35, 1675.

106. Schuda, P. F.; Ebner, C. B.; Morgan, T. M.; Tetrahedron Lett. 1986, 27, 2567.

107. Haefliger, W.; Hauser, D.; Synthesis 1980, 236.

108. Cherif, S. E.; René, L.; Synthesis 1988, 138.

109. Baraldi, P. G.; Barco, A.; Benetti, S.; Manfredini, S.; Pollini, G. P.; Simoni, D.; Tetrahedron Lett. 1984, 25, 4313.

110. Gallos, J. K.; Koftis, T. V.; J. Chem. Soc., Perkin Trans. 1 2001, 415.

111. Valduga, C. J.; Santis, D. B.; Braibante, F. S.; Braibante, M. E. F.; J. Heterocycl. Chem. 1999, 36, 505.

112. Baranovsky, A. V.; Groen, M. B.; de Groot, A.; Litvinovskaya, R. P.; Khripach, V. A.; Collect. Czech. Chem. Commun. 1998, 63, 1564.

113. Natale, N. R.; Tetrahedron Lett. 1982, 23, 5009.

114. Fuentes, J. A.; Maestro, A.; Testera, A. M.; Báñez, J. M.; Tetrahedron: Asymm. 2000, 11, 2565.

115. McNab, H.; Monahan, L. C.; J. Chem. Soc., Chem. Commun. 1985, 213.

116. Pommelet, J.-C.; Dhimane, H.; Chuche, J.; Célérier, J.-P.; Haddad, M.; Lhommet, G.; J. Org. Chem. 1988, 53, 5680.

117. Dhimane, H.; Pommelet, J.-C.; Chuche, J.; Célérier, J.-P.; Lhommet, G.; Haddad, M.; Tetrahedron Lett. 1987, $28,885$.

118. Jourdain, F.; Pommelet, J. C.; Tetrahedron Lett. 1994, 35, 1545.

119. Fasseur, D.; Cauliez, P.; Couturier, D.; Rigo, B.; Defretin, S.; J. Heterocycl. Chem. 1994, 31, 829.

120. Fasseur, D.; Cauliez, P.; Couturier, D.; Rigo, B.; Defretin, S.; J. Heterocycl. Chem. 1996, 33, 1951.

121. Basavaiah, D.; Rao, P. D.; Hyma, R. S.; Tetrahedron 1996, 52, 8001.

122. Kim, J. N.; Kim, H. S.; Gong, J. H.; Chung, Y. U.; Tetrahedron Lett. 2001, $42,8341$.

123. Shono, T.; Matsumura, Y.; Tsubata, K.; Sugihara, Y.; Yamane, S.; Kanazawa, T.; Aoki, T.; J. Am. Chem. Soc. 1982, 104, 6697.

124. Aki, O.; Nakagawa, Y.; Chem. Pharm. Bull. 1972, 20, 1325.

125. Barum, O.; Mohanta, P. K.; Ila, H.; Junjappa, H.; Synlett 2000, 653.

126. Molina, P.; Pastor, A.; Vilaplana, M. J.; J. Org. Chem. 1996, 61, 8094

127. Palacios, F.; Herrán, E.; Rubiales, G.; J. Org. Chem. 1999, 64, 6239.

128. Collin, J.; Jaber, N.; Lannou, M.I.; Tetrahedron Lett. 2001, 42, 7405.

129. Olah, G. A.; Iyer, P. S.; Prakash, G. K. S.; Synthesis 1990, 487.

130. Kumareswaran, R.; Reddy, B. G.; Vankar, Y. D.; Tetrahedron Lett. 2001, 42,7493 . 